\title{
ARCHITECTURAL HERITAGE DOCUMENTATION BY USING LOW COST UAV WITH FISHEYE LENS: OTAG-I HUMAYUN IN ISTANBUL AS A CASE STUDY
}

\author{
N. Yastikli ${ }^{\text {a }}$ Ö. Z. Özerdemª \\ ${ }^{a} Y T U$, Civil Engineering Faculty, Department of Geomatics Engineering, 34210 Davutpasa, Istanbul, Turkey - \\ ynaci,@yildiz.edu.tr, omerzubeyr.ozerdem@gmail.com
}

KEY WORDS: Cultural heritage, Documentation, Photogrammetry, SfM, UAV, 3D modelling

\begin{abstract}
:
The digital documentation of architectural heritage is important for monitoring, preserving, managing as well as 3B BIM modelling, time-space VR (virtual reality) applications. The unmanned aerial vehicles (UAVs) have been widely used in these application thanks to rapid developments in technology which enable the high resolution images with resolutions in millimeters. Moreover, it has become possible to produce highly accurate $3 \mathrm{D}$ point clouds with structure from motion (SfM) and multi-view stereo (MVS), to obtain a surface reconstruction of a realistic 3D architectural heritage model by using high-overlap images and 3D modeling software such as Context capture, Pix4Dmapper, Photoscan.
\end{abstract}

In this study, digital documentation of Otag-i Humayun (The Ottoman Empire Sultan's Summer Palace) located in Davutpaşa, Istanbul / Turkey is aimed using low cost UAV. The data collections have been made with low cost UAS 3DR Solo UAV with GoPro Hero 4 with fisheye lens. The data processing was accomplished by using commercial Pix4D software. The dense point clouds, a true orthophoto and 3D solid model of the Otag-i Humayun were produced results. The quality check of the produced point clouds has been performed. The obtained result from Otag-i Humayun in Istanbul proved that, the low cost UAV with fisheye lens can be successfully used for architectural heritage documentation.

\section{INTRODUCTION}

The preservation of the cultural heritage is one of the main issues for all countries. Like other countries, documentation and inventory of the cultural heritage in Turkey needs to be completed as soon as possible for the efficient conservation interventions. In Turkey, approximately 60.000 monumental buildings, 75.000 fixed Cultural Heritage and 8200 protected sites and 2.800.000 movable heritage are registered till now (Çayirezmez, 2007).

The digital photogrammetry has been used successfully for documentation of the cultural heritage. The terrestrial laser scanning system is another tool for architectural heritage documentation as well as $3 \mathrm{D}$ modelling and visualization (Yastikli, 2007). Unmanned Aerial Vehicles (UAVs) serves new opportunities in photogrammetry applications as well as applications in robotics, computer vision, agriculture and environment, surveillance and reconnaissance, aerial monitoring in engineering, cultural heritage (Niethammer et al., 2009; Irschara et al., 2010; Sauerbier et al., 2010). In recent years, UAV systems are frequently used in photogrammetry application because of the possibility of capturing cost effective data at high spatial and temporal resolution. The light UAV platform with amateur or SRL digital cameras and GNSS/INS systems, can be used for photogrammetric data acquisition. The limited pay-load of the UAVs are restrict the transportation of the high quality IMU devices coupled with the airborne cameras or LiDAR sensors used for large scale mapping. The low cost UAV systems are available with the very reasonable price in comparison to the digital photogrammetric systems. The low cost UAV systems can be used as a tool for architectural documentation instead of the terrestrial laser scanning system.
The main aim of this study is to evaluate the performance of the low cost light UAV system with fisheye lens for architectural documentation. Considering this aims, this paper is organized as follows; the next section describes the methodologies which includes the details of proposed approach for automatic point clouds and 3D model generation approaches for architectural heritage documentation. This is followed by the study area, data set and the experimental section which provides the detailed information about performance evaluation of the low cost light UAV system with fisheye lens for architectural documentation. The concluding remarks are given in last section.

\section{METHODOLOGY}

The currently developed software tools are enable to conduct image orientation and dense image matching at the same platform with the acquired image using UAV. The development of high performance, low-cost, user-friendly and innovative 3D modeling software has contributed significantly to the expansion of UAVs in photogrammetry applications. The Structure from Motion (SfM) and/or Multi-View Stereo (MVS) are the most popular approaches and implemented in many 3D modelling software such as Agisoft Photoscan Professional (PS), Pix4D, 3Df Zephyr (3DZ), SURE, MicMac (MM), VisualSFM (VSFM), and ContextCapture (CC).

The images captured by the UAV are used for matching with the $\mathrm{SfM}$ approach based on the common details between image pairs using the SIFT (Scale-Invariant Feature Transform). First, the image orientations have been conducted and then the dense image matching is performed in the $3 \mathrm{D}$ modeling software utilizing computer vision techniques based on SfM. The reconstruction of the 3D space by overlapping the images from different perspectives with SfM approach can be easily generated with high accuracy using computer vision techniques and the

\footnotetext{
* Corresponding author
} 
flight planning in photogrammetry (Yanagi et al., 2016; Nakano et al., 2016).

As mentioned earlier, the main motivation of this study is to test the performance of the low cost light UAV system with fisheye lens. The proposed approach for architectural documentation includes field work and image acquisition, 3D model generation and accuracy assessment. Figure 1 shows the workflow of the proposed strategy for 3D model generation with the low cost light UAV system with fisheye lens.

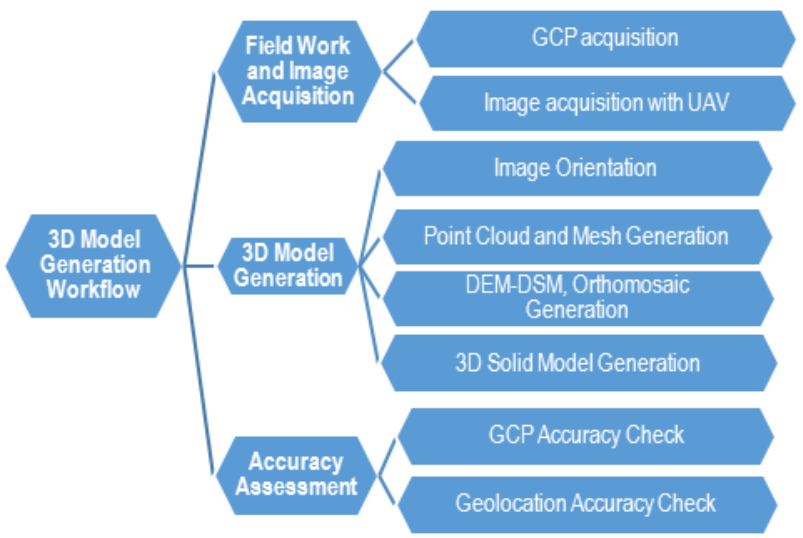

Figure 1. Workflow of 3D model generation of architectural heritage.

\section{STUDY AREA AND DATA ACQUISITION}

\subsection{Study Area}

The Otag-i Humayun (The Ottoman Empire Sultan's Summer Palace) located in Davutpasa Campus of Yildiz Technical University, Istanbul / Turkey (Figure 2) was used as a study area to test the performance of the low cost light UAV system with fisheye lens for digital documentation of architectural heritage.

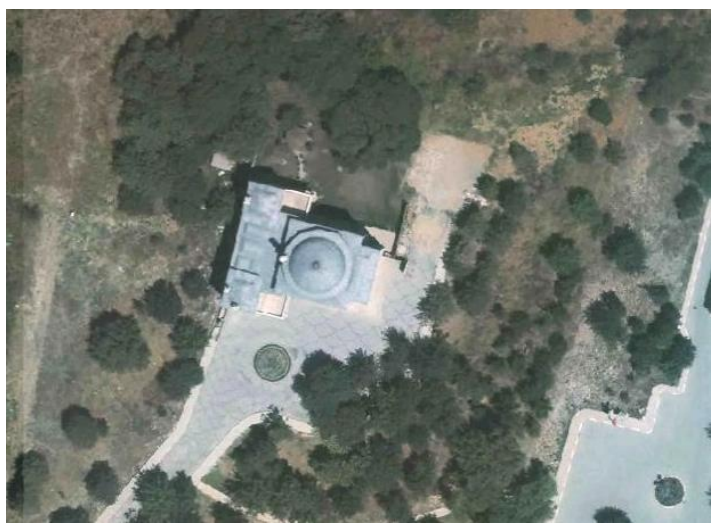

Figure 2. The study area The Otag-i Humayun located in Davutpasa Campus of Yildiz Technical University, Istanbul The Otag-i Humayun building located in Historical Davutpaşa old site was used both military purposes and summer place of the Ottoman Sultan's.

\subsection{UAV Platform}

The low cost UAV used in this study is consist of 3DR Solo rotary-wing quadcopter UAV equipped with GoPro Hero 4 action camera fisheye lens mounted on 3-axis stabilized gimbals, Solo smart controller and mobile android GM5Plus device as second smart controller-GCS. The 3DR Solo can automatically control both copter and camera positioning in flight. The selected the GoPro Hero 4 action camera for image acquisition was optimized for Solo the lightweight, fisheye lens. Table 1 shows the 3DR Solo Multicopter specifications used in this study.

\begin{tabular}{|l|l|}
\hline Item & Specifications \\
\hline Rotor & 4 \\
\hline Flight controller & $\begin{array}{l}\text { 3D Robotics PixHawk } \\
\text { APM fully-autonomous FC } \\
\text { software }\end{array}$ \\
\hline Flight log & Recordable \\
\hline Battery & $\begin{array}{l}\text { Lithium polymer 6-cell 2 } \\
5300 \text { mAh }\end{array}$ \\
\hline Camera & GoPro HERO4 Black Edition \\
\hline Weight & ca. 1800 g \\
\hline Flight time & ca. 20 min \\
\hline Autonomous flight & Leading waypoints \\
\hline GNSS & $\begin{array}{l}\text { Ublox Neo-M8N GPS PPM } \\
\text { OSD 3DR 915-433Mhz }\end{array}$ \\
\hline
\end{tabular}

Table 1. 3DR Solo Multicopter specifications

\subsection{Data Acquisition}

The small double grid block was planned for flight using the open source Pix4DCapture software with mobile GM5Plus device. The planned block which consist of 28 images including three flight strips with $90 \%$ endlap in flight direction and $80 \%$ sidelap for traditional nadir images acquisitions. The 5 ground control point (GCP) target called Pix4Dtagger GCP targets were placed on the study area near the Otag-i Humayun building. The GCP target was a black-and-white target with the dimensions $100 \mathrm{~cm}$ $\times 100 \mathrm{~cm}$ (Figure 3). The local coordinates of the GCP targets were measured and converted into the geodetic coordinates using a GNSS (South Surveying \& Mapping Instrument, SOUTH S82V) with the accuracy of $\pm 3 \mathrm{~mm}$.

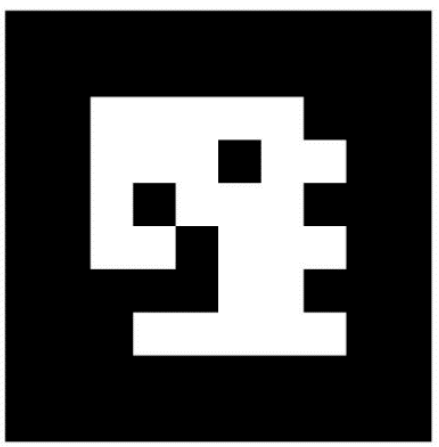

Figure 3. The GCP target (Pix4Dtagger GCP target)

The planned fight (Figure 4) was performed on 6 April 2017 over study area at a height of $50 \mathrm{~m}$ with the GoPro Hero 4 action camera (12 Mpx and pixel size $1.44 \mu \mathrm{m})$ with $3.00 \mathrm{~mm}$ focus length fisheye lens at the ground sample distance (GSD) of 3.1 $\mathrm{cm}$. 


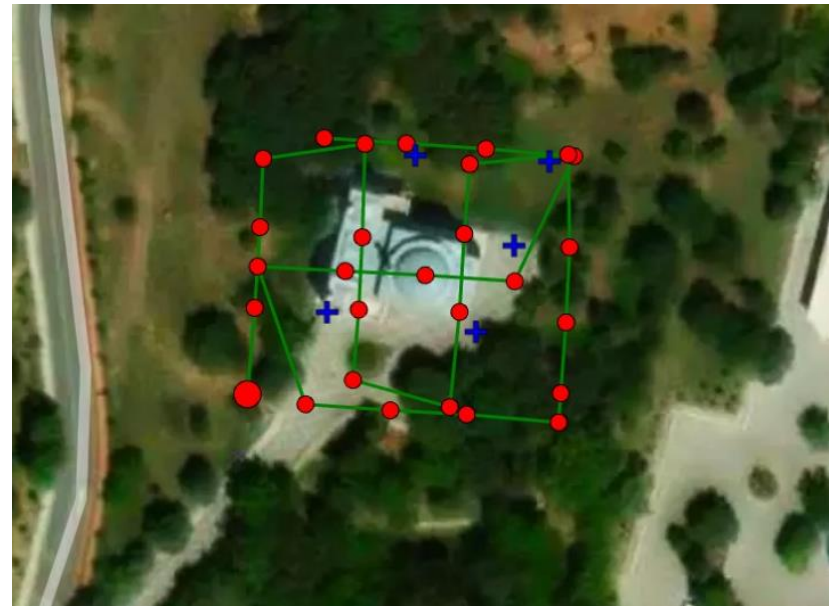

Figure 4. The flight plan and distribution of the GCP on the study area

\section{PHOTOGRAMMETRIC DATA PROCESSING}

The photogrammetric data processing starts with the determination of the geotagging flight information and camera parameters for each recorded image during the image acquisition. Geotagging steps add geographical locations to each image. This step called the determination of interior and exterior parameters of the image in photogrammetry. The measured coordinate of the GCPs is used in camera calibration to the geometric calibration parameters including the position of principal point and focal length ( $\mathrm{x} 0, \mathrm{y} 0, \mathrm{c})$, radial distortion $(\mathrm{K} 1, \mathrm{~K} 2, \mathrm{~K} 3)$ and tangential distortion $(\mathrm{P} 1, \mathrm{P} 2)$ parameters. The $3 \mathrm{D}$ point cloud generation is the next steps with dense image matching algorithm to reconstruct 3D model. All these steps can be accomplished by fully automated process with available commercial software tools. In this study, four software packages were employed;

- Pix4DCapture for image acquisition,

- Pix4Dtagger to capture GCP on images automatically (with square code),

- Pix4Dmapper for 3D point cloud generation from stereo images,

- Autodesk ReCap for 3D solid model generation.

The photogrammetric data processing steps for generation of 3D Model are given as follows;

- Initial Processing (Automatic Aerial Triangulation)

- Keypoint extraction and matching,

- Building automatic tie points,

- Computing matches,

- Camera calibration and optimization,

- Point Cloud and Mesh Generation

- Dense matching

- Constrain point cloud

- Surface Reconstruction

- Dense mesh creation, decimation, improving, segmentation, texture coordinates,

- Exporting 3D Mesh,

- DEM-DSM, Orthomosaic Generation

- Generation of color point cloud

- Generation of orthomosaic

- Generation of orthocolor gridded DSM

- 3D Solid Model Generation

- Generation of 3D solid model

- Generation of animation.
As results of the photogrammetric data processing steps, the generated 3D point cloud, DSM and orthomosaic, and 3D solid model of the study area are given in Figure 5, Figure 6 and Figure 7 respectively.

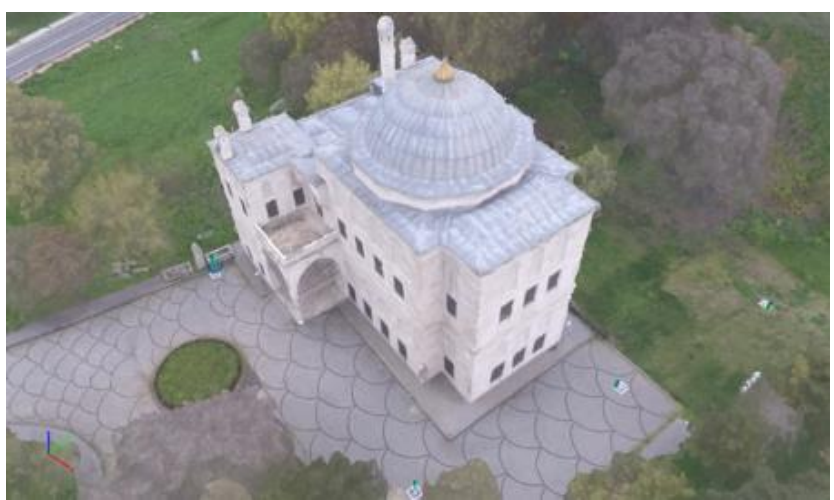

Figure 5. The generated 3D point cloud of the study area

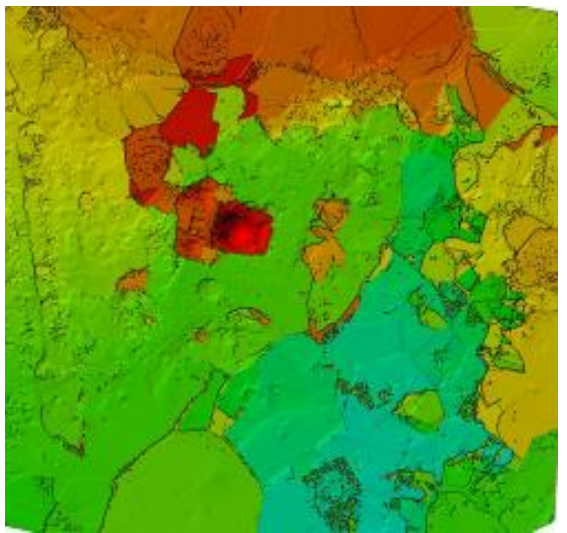

(a)

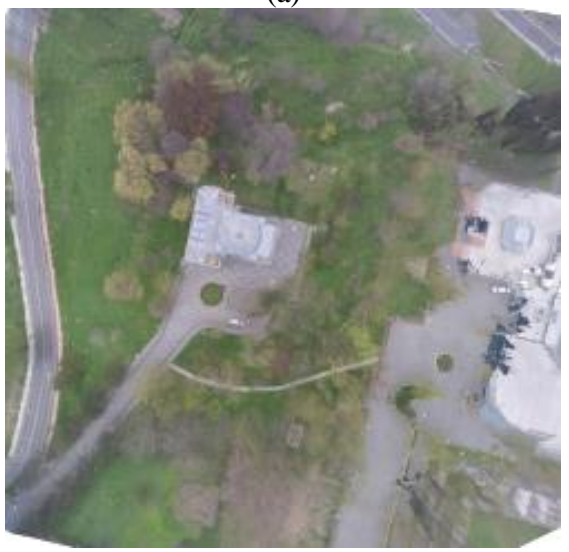

(b)

Figure 6. The generated DSM (a) and orthomosaic (b) of the study area 


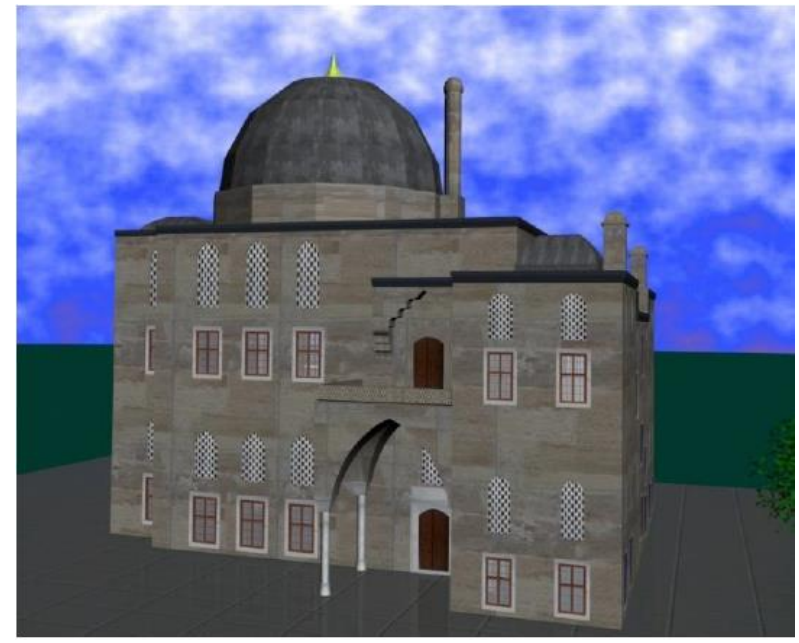

Figure 7. The generated 3D solid model of the study area

During the data processing, the UAV path planning SLAM (simultaneous localization and mapping) and PPK (Post Processed Kinematic) with GNSS (RINEX) data were used to achieve high accuracy for determination of the geotagging flight information and camera parameters for each recorded image. The internal accuracy check was performed using the Pix4Dmapper software package and the reached results were better than expected accuracy for $\mathrm{X}, \mathrm{Y}$ and $\mathrm{Z}$ coordinates.

The very dense point cloud of study area was produced with the average density of 791 points per $\mathrm{m}^{3}$ including the dens point on facades of the Otag-i Humayun. The recorded 28 nadir images with fisheye lens successfully calibrated used for complete 3D modeling of the architectural heritage including terrain, trees, historical building facades and roofs. As can be seen in Figure 5, Figure 6 and Figure 7, the 3D point cloud, DSM, orthomosaic and 3D solid model of the The Otag-i Humayun located in Davutpasa Campus of Yildiz Technical University, Istanbul were successfully generated with the low cost UAV with fisheye lens.

\section{CONCLUSIONS}

In this study, the performance of the low cost 3DR Solo rotarywing quadcopter UAV equipped with GoPro Hero 4 action camera fisheye lens mounted on 3-axis stabilized gimbals, Solo smart controller was tested for digital documentation of Otag-i Humayun as an architectural heritage located in Davutpaşa, Istanbul as study area.

The small double grid image block which consists of 28 nadir images including three flight strips with $90 \%$ endlap in flight direction and $80 \%$ sidelap was planned and performed on 6 April 2017. The photogrammetric data processing have been conducted using the Pix4D software and 3D point cloud, DSM and orthomosaic generation has been performed and The 3D solid model was generated with Autodesk ReCap. All recorded nadir images with fisheye lens successfully calibrated used for complete 3D of the architectural heritage documentation. The 3D point cloud including the point on terrain, trees, historical building facades and roof was successfully generated as well as DSM, orthomosaic and 3D solid model of the The Otag-i Humayun.

The UAV systems frequently used in photogrammetry application especially documentation architectural heritage because of its ability to quick deliver high temporal and spatial resolution image information. The derived high-resolution images with UAV can be used for geometric modeling, generation of map and mosaic, stereo drawing, 3D modelling and texture mapping on existing 3D data of architectural heritage. Rotary wing UAV platforms can even take-off and land vertically without any requirement for runway area and records the nadir oblique and nadir images at the same time. With the available commercial software package, all data processing can be performed almost automatically.

The stability and endurance of low-cost light UAV system for is open issue architectural heritage documentation. Because of that, the performance test of low cost light UAV system for digital documentation of Otag-i Humayun as an architectural heritage was aimed in this study. The obtained results from our test side (Otag-i Humayun) verified that, the low cost light UAV system with fisheye lens can be used successfully for architectural heritage documentation with the limited number of nadir images without any oblique images. The dense 3D point cloud, DSM, orthomosaic and 3D solid model of Otag-i Humayun were successfully generated with proposed work follow.

\section{REFERENCES}

Cayirezmez, N., A., 2007. Cultural Heritage Inventory System of Turkey on the Web, Int. Arch. Photogramm. Remote Sens. Spatial Inf. Sci., XXXVI-5/C53, /isprs-archives- XXXVI-5/C53, 2007.

Irschara, A., Kaufmann, V., Klopschitz, M., Bischof, H., Leberl, F., 2010. Towards fully automatic photogrammetric reconstruction using digital images taken from UAVs. Proc. ISPRS Symposium, 100 Years ISPRS - Advancing Remote Sensing Science.

Nakano, K., Suzuki, H., Tamino, T., Chikatsu, H., 2016. On Fundamental Evaluation Using UAV Imagery and 3D Modeling Software. Int. Arch. Photogramm. Remote Sens. Spatial Inf. Sci., XLI-B5, 93-97, https://doi.org/10.5194/isprs-archives-XLI-B593-2016.

Niethammer, U., Rothmund, S. and Joswig, M., 2009. UAVbased remote sensing of the slow moving landslide Super-Sauze. In: Landslide processes, CERG Editions, Strasbourg, pp. 69-74.

Sauerbier, M., Eisenbeiss, H., 2010. UAVs for the documentation of archaeological excavations. IAPRS \& SIS, Vol. 38(5), Newcastle upon Tyne, UK.

Yanagi, H., Chikatsu, H., 2016. Performance Evaluation of 3D Modeling Software for UAV Photogrammetry. Int. Arch. Photogramm. Remote Sens. Spatial Inf. Sci., XLI-B5, 147-152, https://doi.org/10.5194/isprs-archives-XLI-B5-147-2016, 2016

Yastikli, N. (2007). Documentation of cultural heritage using digital photogrammetry and laser scanning. Journal Of Cultural Heritage, 8(4), 423-427. 